\title{
Calibration Monitor for Dark Energy Experiments
}

\author{
DE-FG02-07ER41506
}

\section{Final Scientific/Technical Report}

This report contains no distribution restrictions.

\author{
Submitted by: Dr. Mary Elizabeth Kaiser \\ Principal Investigator \\ Address: The Johns Hopkins University \\ Department of Physics and Astronomy \\ 3400 North Charles Street \\ Baltimore, MD 21218 \\ 410-516-5088 \\ kaiser@pha.jhu.edu
}




\section{Project Information}

DOE Grant Award Number:

Recipient Institution:

Address:

Principal Investigator:

Project Title:

DOE/Office of Science Program Office:

DOE/Office of Science Program Office

Technical Contact:

\section{DE-FG02-07ER41506}

The Johns Hopkins University

Department of Physics and Astronomy

3400 North Charles Street

Baltimore, MD 21218

410-516-5088

kaiser@pha.jhu.edu

Dr. Mary Elizabeth Kaiser

Calibration Monitor for Dark Energy Experiments

High Energy Physics

Dr. Kathleen Turner

The pont-of-contact for the collaboration is the P.I., Dr. Mary Elizabeth Kaiser.

\begin{tabular}{llll}
\hline \hline Project Team Member & & Affiliation & \\
\hline \hline & & & \\
Mary Elizabeth Kaiser & PI & JHU $^{1}$ & ${ }^{1}$ The Johns Hopkins University \\
Stephan R. McCandliss & Co-I & JHU $^{1}$ & \\
Jeffrey W. Kruk & Co-I & JHU $^{1}$ & \\
Ralph C. Bohlin & Co-I & STScI $^{2}$ & ${ }^{2}$ Space Telescope Science Institute \\
Susana E. Deustua & Co-I & AAS $^{3}$ & ${ }^{3}$ American Astronomical Society \\
W. Van Dyke Dixon & Co-I & JHU $^{1}$ & \\
Randy A. Kimble & Co-I & GSFC $^{4}$ & ${ }^{4}$ Goddard Space Flight Center \\
Bernard J. Rauscher & Co-I & GSFC $^{4}$ & \\
David J. Sahnow & Co-I & JHU $^{1}$ & \\
Paul D. Feldman & Collaborator & JHU $^{1}$ & \\
Jonathan P. Gardner & Collaborator & GSFC $^{4}$ & \\
Robert Kurucz & Collaborator & CfA $^{5}$ & ${ }^{5}$ Harvard Smithsonian Center for Astrophysics \\
Michael Lampton & Collaborator & SSL $^{6}$ & ${ }^{6}$ Space Sciences Laboratory, U.C. Berkeley \\
H. Warren Moos & Collaborator & JHU $^{1}$ & \\
Saul Perlmutter & Collaborator & UCB $^{7} / \mathrm{LBNL}^{8}$ & ${ }^{7}$ University of California, Berkeley \\
& & ${ }^{8}$ Lawrence Berkeley National Laboratory \\
Adam Riess & Collaborator & JHU $^{1} / \mathrm{STScI}^{2}$ & \\
Bruce E. Woodgate & Collaborator & GSFC $^{4}$ & \\
Edward L. Wright & Collaborator & UCLA $^{9}$ & ${ }^{9}$ University of California, Los Angeles \\
\hline \hline
\end{tabular}




\section{Calibration Monitor for Dark Energy Experiments}

\section{Executive Summary}

\subsection{Overview}

The goal of this program was to design, build, test, and characterize a flight qualified calibration source and monitor for a Dark Energy related experiment: ACCESS - "Absolute Color Calibration Experiment for Standard Stars".

This calibration source, the On-board Calibration Monitor (OCM), is a key component of our ACCESS spectrophotometric calibration program. The OCM will be flown as part of the ACCESS sub-orbital rocket payload in addition to monitoring instrument sensitivity on the ground. The objective of the OCM is to minimize systematic errors associated with any potential changes in the ACCESS instrument sensitivity. Importantly, the OCM will be used to monitor instrument sensitivity immediately after astronomical observations while the instrument payload is parachuting to the ground. Through monitoring, we can detect, track, characterize, and thus correct for any changes in instrument senstivity over the proposed 5-year duration of the assembled and calibrated instrument.

\subsection{How the Project Contributes to the Field of Research}

The OCM is a feedback stabilized light source that will be flown as part of the ACCESS instrument. It will provide data on the instrument stability immediately after the astronomical observations have been performed. This provision to provide feedback on instrument performance at the time of observation constrains systematic errors and is the reason why the OCM is an essential element of the ACCESS instrument whose goal is to provide precise measurements of stellar sources to enable the establishment of an improved astrophysical flux scale.

ACCESS (Kaiser et al., 2008, 2009) is a series of rocket-borne sub-orbital missions and groundbased experiments whose primary purpose is to provide the first step in the fundamental color calibration required by SNeIa observations obtained in pursuit of understanding the nature of Dark Energy (DE). The ACCESS program is designed to obtain an absolute spectrophotometric calibration accuracy of $<1 \%$ in the $0.35-1.7 \mu \mathrm{m}$ bandpass at a spectral resolution greater than 500 by directly tracing the observed stellar fluxes to National Institute of Standards and Technology (NIST) irradiance standards. The absolute calibration of the telescope and spectrograph will be performed to obtain direct measurement of the absolute fluxes in physical units using calibrated detectors as the fundamental metrology reference. These flux measurements will also place the existing networks of standards on an improved absolute scale and make them available to all telescopes enabling their use as standard sources.

To achieve this goal, a series of ground-based tasks needs to be completed. The design, fabrication and characterization of a calibration monitoring system, the OCM, is one of these tasks.

\subsection{Project's Specific Relevance to Dark Energy Programs}

The OCM program contributes to SNeIa based dark energy investigations, by enabling the establishment a high-precision photometric and spectrophotometric calibration system in the ultraviolet, visible, and near-infrared. Increasing the precision in the visible through nearinfrared spectrophotometric calibration system is important for flux-based astronomical measurements and specifically for the determination of precision photometric redshifts, K-corrections, and luminosity distances in SNeIa based Dark Energy measurements by minimizing one of the systematic errors that can reduce the precision of these results.

The OCM is a vital component in achieving this increased precision in the spectrophotometric calibration by enabling knowledge/monitoring of the instrument sensitivity from the moment the instrument payload is calibrated to immediately after the observation is executed.

\subsection{Project Design and Technical Effec- tiveness}

The OCM system tracks the instrument sensitivity by providing a feedback stabilized light source to the instrument. This system consists of an illuminating source, a current feedback controller, and an optical transfer system to deliver a uniform illumination to the telescope (Fig. 1).

The illuminating source is a ring of LEDs pairs. Each pair has a unique central wavelength and the ensemble of LEDs spans the wavelength range of the instrument. Each LED is monitored by a photodiode that provides input to the current feedback controller and results in a constant 
light output from the LED. The optical transfer system consists of a diffuser mounted on the interior of the telescope cover. The stable illumination of the diffuser provides the source that will be monitored during the absolute ground calibration, in the field prior to launch, and in-flight.

This program has resulted in the (1) measurement of the temperature dependance of the flux output of the LEDs, (2) the measurement of LED brightness as a function of current, (3) the selection of a spectrally uniform diffuser material (Frostkote) to be mounted inside the telescope cover as part of the OCM, (4) the measurement of a sample of LEDs to select for spectral, spatial, and brightness suitability, (5) detailed spectral measurement of LEDs, (6) selection of monitoring photodiodes, and (7) the design of the OCM electronics and feedback system based on the LED and photodiode measurements performed.

\subsection{Project Accomplishments}

This program has proven the feasibility of the original concept of illuminating a diffuser mounted inside the telescope cover/shutter with a ring of feedback stabilized LEDs located near the telescope secondary mirror.

The tests and measurements outlined in the previous section enabled the selection of LEDs, feedback photodiodes, diffuser material, and design of the feedback electronics and circuit boards and procurement of the flight unit.

The instrument payload is currently being fabricated. The final implementation and test of the flight unit will occur during payload integration and test. As a result of the tests and measurements performed in the lab as part of this program, dual stabilization of the LEDs on temperature and brightness may be necessary for the flight unit. Consequently, the flight unit is awaiting the determination of the base operating temperature. Once this has been determined the unit will be delivered, integrated, and the final tests performed.

The results of these tests have been published as conference proceedings. The results of the integrated assembly will be published when the payload is completed and those tests performed.

\subsection{Project Activities}

The program consisted of building a test device to measure the brightness ofthe LEDs as a function of temperature and current. In addition, a prototype was built to determine the in-

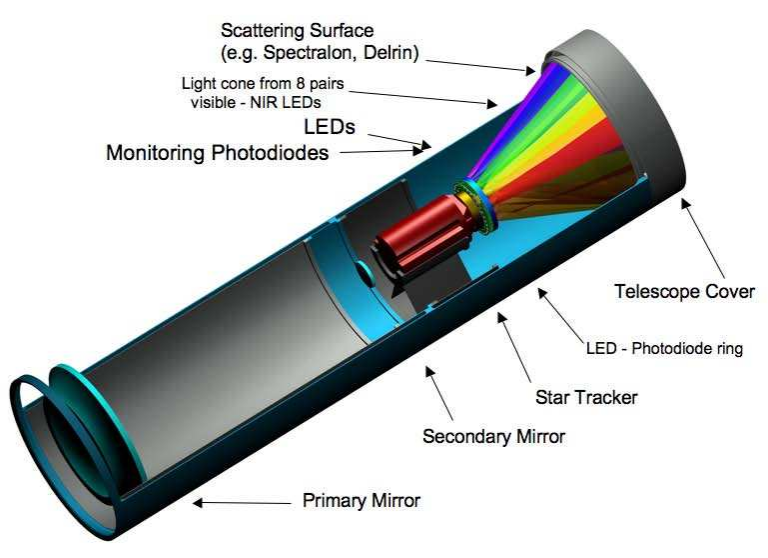

Figure 1: The OCM LEDs are mounted in an annular ring about the star tracker behind the secondary mirror and they illuminate a diffuser on the inside of the telescope cover which in turn illuminates the telescope primary with an angular distribution of rays.

tensity and spatial uniformity of the LED output and the reflective (spatial and spectral) uniformity diffuser material. This required integrating an xy-stage to the telescope prototype to obtain measurements. A monochromator system was used to measure the spectral distribution of the LEDs and select LEDs that would provide adequate bandpass coverage.

The dependence of both the central wavelength and brightness of the LED output on temperature (e.g. Figs. 2 \& 3) may result in implementation of an additional control component (temperature in addition to brighness feedback).

\subsection{Publications}

The design and mesurements of the OCM were presented at two conferences, the SPIE Astronomical Instrumentation Conference, Marseille France, June 23, 2008, sponsored by SPIE, the Society of Photo-Optical Instrumentation Engineers (Kruk et al., 2008) and at the CALCON Technical Conference on Characterization and Radiometric Calibration for Remote Sensing, Logan, Utah, August 24, 2009 sponsored by the Space Dynamics Laboratory and Utah State Research Foundation (Kaiser et al., 2009).

Both conference proceedings publications are included with this technical report.

\section{References}

Kaiser, M. E., J. W. Kruk, S. R. McCandliss, D. J. 


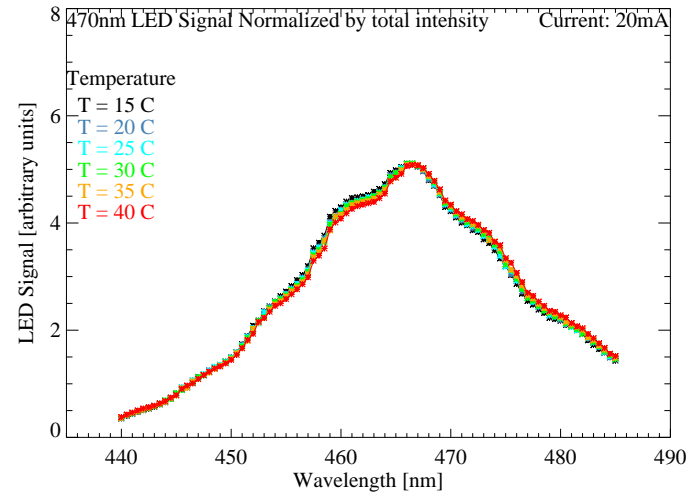

Figure 2: The spectral energy output of the LED as a function of temperature is shown for the $470 \mathrm{~nm}$ LED. The total intensities have been normalized to common values, representing the effect of feedback control Kruk et al. (2008).

Sahnow, R. H. Barkhouser, W. V. Dixon, P. D. Feldman, H. W. Moos, J. Orndorff, R. Pelton, A. G. Riess, B. J. Rauscher, R. A. Kimble, D. J. Benford, J. P. Gardner, R. S. Hill, B. E. Woodgate, R. C. Bohlin, S. E. Deustua, R. Kurucz, M. Lampton, S. Perlmutter, and E. L. Wright 2009. ACCESS: Enabling an Improved Flux Scale for Astrophysics. $18^{\text {th }}$ Annual CALCON Technical Conference.

Kaiser, M. E., J. W. Kruk, S. R. McCandliss, D. J. Sahnow, B. J. Rauscher, D. J. Benford, R. C. Bohlin, S. E. Deustua, W. V. Dixon, P. D. Feldman, J. P. Gardner, R. A. Kimble, R. Kurucz, M. Lampton, H. W. Moos, S. Perlmutter, A. G. Riess, B. E. Woodgate, and E. L. Wright 2008. ACCESS: absolute color calibration experiment for standard stars. Volume 7014 of SPIE Conference Series.

Kruk, J. W., M. E. Kaiser, S. R. McCandliss, J. Orndorff, R. H. Barkhouser, D. J. Sahnow, D. J. Benford, R. C. Bohlin, S. E. Deustua, W. V. Dixon, P. D. Feldman, J. P. Gardner, R. A. Kimble, R. Kurucz, M. Lampton, H. W. Moos, S. Perlmutter, B. J. Rauscher, A. G. Riess, B. E. Woodgate, and E. L. Wright 2008. On-board Calibration Monitor for Tracking Instrument Sensitivity. In S. 7014 (Ed.), Society of Photo-Optical Instrumentation Engineers (SPIE) Conference Series: Astronomical Instrumentation, Volume 7014 of SPIE Conference Series, Bellingham, Washington, pp. 115. SPIE.

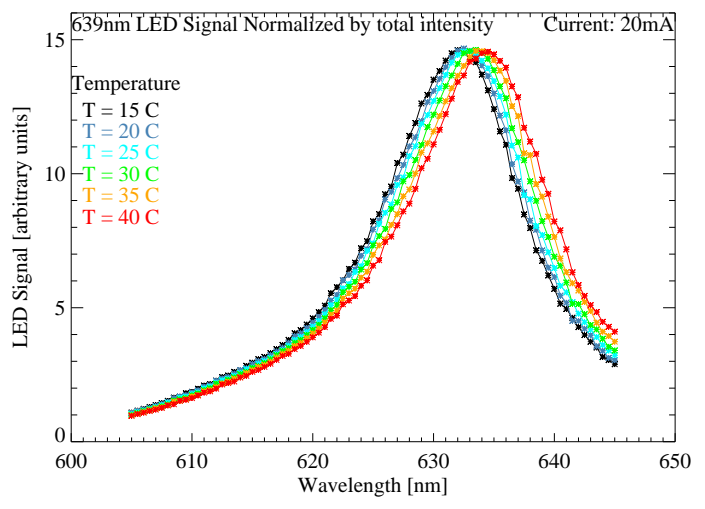

Figure 3: The spectral energy output of the LED as a function of temperature is shown for the $639 \mathrm{~nm}$ LED. The total intensities have been normalized to common values, representing the effect of feedback control Kruk et al. (2008). 